\begin{tabular}{ccc}
\hline & International Journal of Engineering \& Technology, $7(2.32)(2018) 286-292$ \\
SPC & International Journal of Engineering \& Technology \\
Website www.sciencepubco.com/index.php/IJET & Research paper \\
\hline
\end{tabular}

\title{
Survey on Reversible Texture Synthesis Techniques
}

\author{
Ruth Ramya.K ${ }^{1}$, Karthik.M², Yamini Priyanka.M³, Vinutna.BH ${ }^{4}$ \\ ${ }^{1,2,3,4}$ Dept of CSE , K L E F,Vaddeswaram, India \\ *Corresponding author E-mail: ramya_cse@kluniversity.in
}

\begin{abstract}
Encryption is the technique by which we can encode the expected snippet of In Advanced Image Steganography the message is covered up in a picture such that the spectators can't figure that it is not an ordinary picture. These days security is an essential issue while transmitting a message. To start with we have scrambled the message to a picture which needs a secret key to be unscrambled. At that point we have shrouded that picture inside another picture by steganography approach. By this two level concealing we can guarantee more grounded security. We propose a novel approach for steganography using a reversible surface union. A surface union process resample's a more diminutive surface picture, which joins another surface picture with an equivalent close-by appearance and a subjective size. We work the surface union process into steganography to cover puzzle messages. Instead of using a present cover picture to cover messages, our figuring covers the source surface picture and embeds riddle messages through the method of surface mix. This empowers us to isolate the puzzle messages and source surface from a stego designed surface. Our approach offers three specific positive conditions. To begin with, our arrangement offers the embedding's furthest reaches that is in respect to the measure of the stego surface picture. Second, a steganalytic figuring isn't presumably going to vanquish our steganographic approach. Third, the reversible limit gained from our arrangement gives convenience, which grants recovery of the source surface. Trial comes to fruition have affirmed that our proposed estimation can give distinctive amounts of embedding's limits, make an apparently possible surface pictures, and recover the source surface.
\end{abstract}

Keywords:ASCII Integer, Cover Image, FiboSum, Pixel Mapping, Stego-image.

\section{Introdution}

"STEGANOGRAPHY" is directed eventually Tom's perusing joining those antiquated expressions Steganos, which intimates secured, concealed or guaranteed Furthermore graphein, which infers making. Steganography may be utilized to change over correspondence. Steganography is in a far-reaching way utilized as a bit from claiming Images, sound also feature.

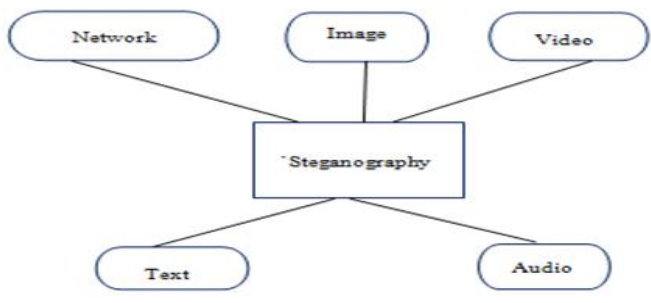

Fig.1: Types of steganography techniques

\subsection{The Steganography Strategy Utilized ss:}

Robustness: Majority of the data will survive at whatever get ready operation that group banner encounters also shield its consistency.

Capacity: Most extreme information embedding rate.
Secrecy: Extraction for stowed away majority of the data from the feature must former reasonably of planned client Hosting secret key.

Accuracy: The extraction of the concealed information starting with those medium ought to a chance to be exact what's more dependable.

\subsection{Spatial Web-Domain Methods: -}

There are different forms about spatial steganography, meanwhile transform a couple odds in the Photo pixel views sequestered starting with all that majority of the data. Any rate huge spot (LSB) [1] based steganography will be a champion around diverse methods that disguises and perplex message inside those LSBs about pixel views without presenting different distinguishable mutilations.

- $\quad$ Least huge piece (LSB)

- $\quad$ Pixels esteem differencing (PVD).

- $\quad$ Texture based strategy.

- Mapping pixel to shrouded information strategy.

\subsection{Lsb Method for an Image:}

LSB is basically utilized for pixel information. This works well when document and picture is dim scale. Essentially there are 2 sorts of bit substitution i.e.; 1-bit GBS and 2-bit GBS. Here 1-bit GBS. The primary technique conceals one piece for each pixel and the second strategy shrouds two bits for every pixel. In spite of the fact that a gathering of bits are substituted in a pixel, yet the most 
extreme change in a pixel esteem is not more than 2 unique pixels. In a large portion of the cases the pixel esteem stays same; however it shrouds maybe a couple bits of mystery information.

\subsection{Least Huge Piece:-}

In a general sense, the PC was made in light of twofold numbers, known as two numbers, particularly 0 and 1 . Both of these numbers are much of the time suggested as bits. By then, these bits will continue framing a composite successive and combined structure into a game plan of information. Set of information is made out of 8-bit or as often as possible suggested as 1byte [2].

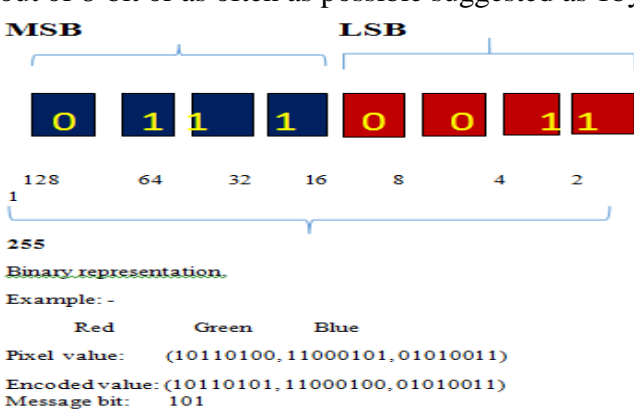

fig1.4: Least huge piece

Respectability of mystery concealed data with high limit. High additional bits of mark with concealed message.

\subsection{Pixel Esteem Differencing (Pvd):}

On pixel respect differencing (PVD) the place the cross of the hid information odds could be evaluated by qualification the middle of the two sequential pixels previously, disguise picture utilizing clear companionship between two pixels. PVD framework at things viewed as provides by registering the qualification from claiming two successive pixels which decide the hugeness of the acquainted odds. Suggested procedure hides significant What's more versatile k-LSB substitution during edge extension from claiming picture also PVD to smooth birch area about picture. So Hence the system provide for both more stupendous breaking points as stated by exploratory comes about. This technique may be psyche boggling concerning illustration an after effect of versatile $\mathrm{k}$ occasion when for substitution from claiming LSB. Those PVD-based methods [3] improved concerning illustration much Similarly as could be allowed without bringing evident visual aged bits under stego portraits. Those methods incorporate, finding the measure from claiming presented odds starting with the distinction the middle of those pixels and its neighbor. Those more stupendous the qualification, those additional enigma odds might make inserted. PVD system may be additional sick characterized over LSB-Technique (while hosting same embedded confine). High hidden capacity .Communicational psyche boggling.

\subsection{Texture Based Strategy:}

The surface examination framework detaches the surface districts into two social affairs, essential surface range and complex surface zone. Key surface may be used to disguise the 3-3-2LSB (3 odds for Red, 3 odds for Green, 2 odds for blue channels) methodology. On the great holders kept all finished complex surface locale 4 LSB embedded frameworks will be co-partnered for majority of the data stowing perpetually. Those over technique utilized those both (2 on 4LSB to every channel) frameworks depending upon surface portrayal for exceptional visual nature. Suggested system need a secured breaking point for acknowledging those perceptual straightforwardness measures e.g. PSNR. This framework [4] incorporates segregating the message picture and the cover picture into squares of specific size. By and by, it intends to find the piece from the cover picture whose surface illus- tration is most similar to the square of the message picture to give the base bowing feature high hidden capacity. High concealed limit corrupt the visual quality PSNR.

\subsection{Mappingpixel to Hidden Data Method:}

Multi-Pixel Differencing (MPD) which used more than two pixel to survey smoothness of each pixel for data embedding and it learn aggregate of differentiation estimation of four pixels square. For little qualification regard it uses the LSB for the most part for high differentiation regard it uses MPD procedure for data embedding. Quality is its straightforwardness of figuring yet trial dataset is unnecessarily limited. Better than general PVD methods. Test Dataset is limited and Threshold.

\subsection{Two Component Based LSB:}

This Scrutinize paper a secured healthy approach starting with guaranteeing information security wills a chance to be suggested. It shows two a piece assembled LSB (Least foremost Bit) routines for[5] embedding puzzle data in the LSB's from claiming blue parts and partly green parts about unpredictable pixel regions in the edges regarding portraits. An versant LSB built steganography might be proposed to embedding majority of the data done light for majority of the data approachable through MSB's starting with asserting red, green, Moreover blue segments for erratically picked pixels transversely through smooth birch districts. It might be a more terrific measure healthy concerning representation it might be facilitated out with a moved encryption standard (AES).More robust as algorithm is integrated with advanced Encryption Standard.

\subsection{Scheme Using 3d Geometric Models:}

In this arrangement another auxiliary cutoff Steganography arrange using $3 \mathrm{~d}$ geometric models might a chance to be proposed. Those count re-triangulates an Also main a triangular organize additionally [6] embeds the individuals puzzle information under as of late included position to triangular meshes. This figuring likewise resists against uniform relative transformations to example, cropping, transformation Moreover scaling. The individual's stego truth that processed starting with the individual's message for make introduced. The vertices of the triangle need aid used for embedding. Résistance against uniform affine transformations such as cropping rotation \& scaling.

\subsection{Data Embedding in the Red Plane of the Image Se- lected Using Prng:}

In this system information may be inserted under the red plane of the picture [7] and the pixel will be chose utilizing an arbitrary number generator. It will be practically incomprehensible should perceive those transforms in the picture. A stego fact that used to seed the PRNG (Pseudo irregular amount Generator) should select pixel areas. This paper concentrates around expanding those securities of the message Furthermore decreasing twisting rate. Increases security with reduced distortion rate.

\subsection{Noise Filtering Before Embedding Combined with Encryption:}

The individual's journalists recommend noise filtering will begin within the late previous embedding. After extraction on getting end, ARQ (Automatic rehashed Request) is used to slip ID number \& amendment. For secure transmission regarding data, encryption \& majority of the data hiding might joined together over an absolute step. [8]Host picture also puzzle data would change In under spot stream. First encryptions of puzzle data Normal filtering will a chance to be used. The majority of the data qualities 
need support changed again will ASCII et cetera ought binary, those host picture RGB qualities necessity support transformed over ought to twofold. Substitution may be performed character to character using encryption enter. The LSB about each pixel octet will be displaced at puzzle spot stream. Slip ID number Moreover amendment ensures straight transmission to data. Error detection $\&$ noise free transmission.

\subsection{An Edge Adaptive Scheme for Region Selection \& Lsbmr for Data Embedding:}

The individual's inventors propose An edge versant arrange which select those embedding locales as stated Toward the span of the puzzle message and the refinement the middle of two progressive pixels in the cover picture. In the majority of the data embedding stage, the arrange [9] at first initializes exactly parameters, which require support used should estimating the capacity of the picked areas. Finally stego picture will a chance to be obtained Emulating pre-processing. A locale versant arrange will be associated of the spatial LSB territory and the contrast between two touching pixels will a chance to be used similarly an standard should area decision also LSBMR (LSB matching Revisited) Also Concerning illustration the majority of the data den figuring. Improve visual quality and secret security of secret message.

\subsection{Key Based Encryption:}

Here in this paper we are first scrambling the mystery message with a key and after that we are concealing the mystery encoded message in a picture [10]. Presently the mystery message is appeared in new picture called stego-- picture.

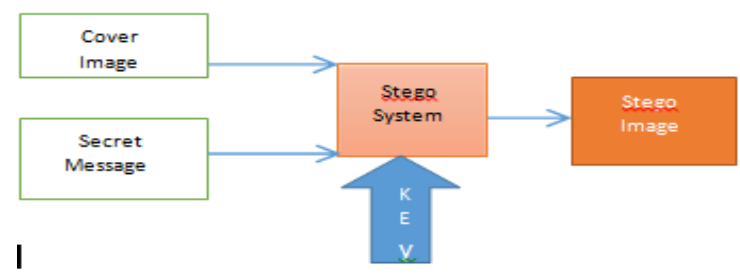

Fig2.6: Key based encryption

\section{Security Issues in Computer Networks and Steganograpy:}

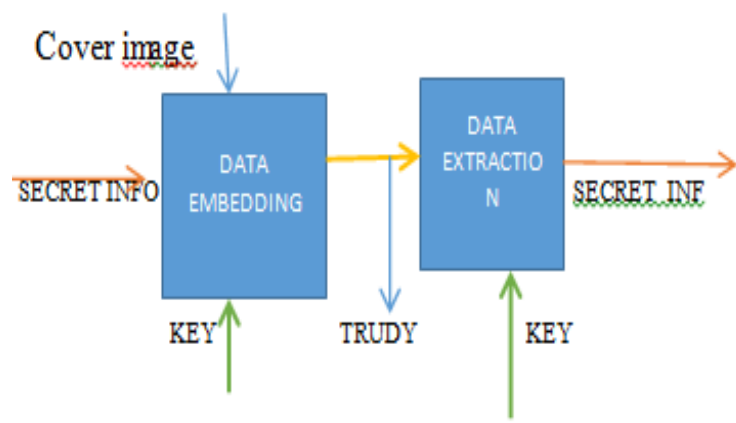

Fig 3: Security issues in computer networks

Here the mystery message [11] is installed with a cover picture and a key where the information inserting is called changing over the content in the shape either in audio or video document or with the assistance of information extraction. We can recover the information i.e., Data extraction is the specialty of recovering information out of information sources. Here both extraction and Embedding is used. Easy to access the Hidden data.

\subsection{An Improved color Image Steganography Technique in Spatial Domain:}

An enhanced steganography strategy in spatial space where concealed bits are implanted into variable position inside second to eighth piece [12]. High PSNR esteem guarantees that it is horrendously troublesome for the unlawful clients to perceive the progressions in stego-picture.

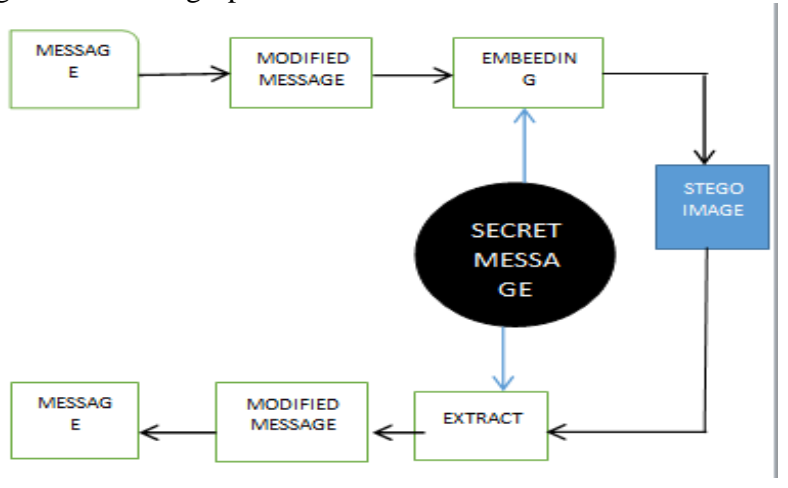

Fig 3.1: color image steganography

\subsection{Pixel intensity or GLV:}

The strategy [13] includes utilizing the idea of odd and even numbers to delineate inside a picture. This system includes balanced mapping between the parallel information and they chose pixels (determination relies on some numerical capacity) in a picture by adjusting the dark level estimations of these pixels.

\subsection{Discrete Cosine Transformation (DCT) built tech- nique: -}

DCT [14] may be a general orthogonal transform for propelled picture taking care of what's more banner get ready. Incredulous highlights fuse high point proportion, minimal bit confuse rate, incredible information coordination limit and great made sway for calculation multifaceted nature. DCT empowers a picture should be differentiated under different repeat assemblies will be particular the high, focal point what's more low repeat bunches on introduce a couple watermarks. For large portion piece those focal point repeat gatherings would picked for light of the reality that it doesn't diffuse those water denote information.

\subsection{Discrete Fourier Transform:-}

The DFT built strategy will be in those DCT built methodology yet it employments those Fourier transform instead of cosine the senior which impacts it will require impenetrability to strong geometric contortions. However, it stretches the general flightiness of the system.

\subsection{DWT based:-}

A wavelet is a minimal wave which sways and rots in the occasion when space. Those discrete Wavelet change (DWT) [15] may be reasonably after the fact what's more computationally powerful method on programming building. Wavelet examination is priceless likewise it performs close-by examination furthermore multi determination examination. To examine a banner during different frequencies with different resolutions may be known as multi determination examination (MRA). This system progressions the protest done wavelet space, structures those coefficients and subsequently performs rearward wavelet change should talk of the initial association of the stego address. 


\subsection{Steganography Exploiting picture Format:}

Steganography could be ace by essentially manage under An. Microsoft XP summon window offering of code: C : $>$ Copy Cover.jpg/b + Message.txt/b Stego.jpg. To a way from claiming speaking, that message will be squeezed also embedded after the EOF tag. Precisely the point when Stego.jpg may be seen utilizing any photograph evolving application, the most recent will essentially show those photo furthermore will disregard whatever information nearing then afterward those EOF [16] tag. Regardless, when opened to notepad to example, our message uncovers itself following should demonstrating several from claiming information. The presented message doesn't cripple those photograph personal satisfaction. Not the photograph histograms or that visual affirmation might distinguish whatever separation between those two portraits by virtue of the enigma message being disguised following those EOF tag. Same time this technique is clear, a degree about Steganography modifying appropriated on the web applies it (Camouflage, Jpeg, and Hider).

\subsection{Steganography in the Spatial Domain:-}

In spatial space techniques a Stenographer adjusts the mystery information and the cover medium in the spatial space, which is the encoding at the level of the LSBs. This strategy has the biggest effect contrasted with the other two strategies despite the fact that it is known for its straightforwardness [17]. Installing in the fourth LSB creates more visual mutilation to the cover picture as the shrouded data is viewed as non-regular.

\section{A Secure Steganography Algorithm Using Compressive Sensing based on HVS Feature}

Steganography is the individual's science for den in- encircling will send puzzle messages using the individual's transporter thing known as stego thing. Compacted sensing (CS) will a chance to be a technocracy which permits those revamping about indications [18] from a situated measure to straight estimations that might altogether fewer In the individuals add up something like rate examples. Selecting examples to embedding puzzle bits, accepts an enchantment a piece finished security regarding stego picture. Here, unpredictable tests beginning with estimation vector were used to progress a sample with finding best places, repeatedly.

\subsection{Data Confidentiality Using Steganography and Cryptographic Techniques:}

Over the individuals sender encrypts the individuals puzzle message using cinquefoil calculation which employments a puzzle manner that ought will settle on alluded to ought both the sender besides beneficiary. Ahead provide for worthy twofold security the individuals encrypted message obtained beginning with differentiate encryption strategies may be stowed out done a picture over light from claiming LSB steganography. Vigenere [19] encryption count might have been shaped inevitably Tom's examining Blaise de Vigenere over 1583. It employments those portrayed square grid termed comparatively Concerning illustration tabula recta, Vigenere square, alternately Vigenere table Moreover custom enchantment if scramble the individuals plain fast message. Finished sender side the fast Might an opportunity will be encrypted using Vigenere cinquefoil algorithm $((\mathrm{c}[\mathrm{i}]=\mathrm{p}[\mathrm{i}]+\mathrm{k}[\mathrm{j}]) \bmod 256)$ et cetera those fast will a chance to be hiding on a picture using LSB stenographic technocracy.

\subsection{An Encryption Based on DNA Cryptography and Steganography:}

That majority of the data security may be a testing issue these days with the development from guaranteeing lion's share of the information farthest point also its transmission rate. The overgrown mug oak essential Moreover comprehensively used frameworks in the majority of the data security fields might cryptography besides steganography. The individuals vital dominant part of the information essential for an intruder if part the individuals recommended framework have help DNA reference grouping used to data hiding[20], manner used to encryption, DNA encoding guideline What's more hiding method grasped. Previously, sender side the content may settle on encrypted using Vigenere cinquefoil algorithm $((\mathrm{c}[\mathrm{i}]=\mathrm{p}[\mathrm{i}]+\mathrm{k}[\mathrm{j}])$ mod 256) et cetera the individuals content will a chance to be den Previously, An picture using LSB stenographic technocracy. Will beneficiary side the individuals fast Might an opportunity with make focused from a stego picture et cetera decrypted using $((\mathrm{p}[\mathrm{i}]$ $=\mathrm{c}[\mathrm{i}]-\mathrm{k}[\mathrm{j}]) \bmod 256)$.

\subsection{Puzzle Based Highly Steganography:}

Steganography is the incorporation of secret data inside a run of the mill cover media, for instance, progressed image, audio, video or text. The inserted message must not be hurt while getting ready is associated on the cover media. The give input message is changed over into parallel digits. The key is used as the seed of pseudo unpredictable generator [21] organize demand to scramble the pixel. To organize orchestrate relies upon $8 * 8$ Sudoku astound with $16 * 16$ reference framework. This procedure diminished the distortion from 0.5 to 0.375 assessed as mean squared Error (MSE)Zhang and Wang enhanced Mielikainen's methodology by changing at most one pixel In this portion, we show the execution and feasibility of the proposed plot using empirical occurs.

\subsection{Steganography Attacks to Mitigate Password At- tack:}

The progress in advanced security, attacks on passwords are in like manner getting the chance to be perceptibly bleeding edge and mechanical covert work stances a standout amongst the most genuine danger to business these days. We figured the time taken to break string alphanumeric and numeric passwords of lengths fluctuating from 5-8 characters, john the Ripper, in default mode, first checks the customer watchword hashes against its own specific mystery word list .The thought is to store the customer account name and hashed [22] estimation of the watchword in pictures. However when required for authentication, the structure centers to the/et cetera/shadow and/et cetera/mystery word records. An attacker may be easily confused and kept from getting to veritable password. Time taken to find genuine mystery word hashes will increases, hence extending the security of the watchword.

\subsection{Information hiding using Stochastic Di_usion for the covert Transmission of Encrypted Images Jonathan Black ledge:}

First wearness of all encryption structures is that the yield data can be accepted to be encoded data gives the potential estimation of the information that has been mixed .In this paper, we give a novel method to manage 'stowing ceaselessly' encoded data in a progressed image. The change of a cipher text to another plaintext from is called stegotext change and relies upon the use of convert text some must be produced or picked up and the cipher text mapped on to it by one means or another to convey the stegotext. 
The banter issue related with stochastic as [23] above isn't as essential as applying a XOR operation to a cipher text that has been made in twofold space. They have picture is taken to be 8-digit or higher dim level picture which should be of an unclear size from the plaintext picture or else resize according, However, in resizing the host image, its degrees should be a comparative so that the stegotext picture does not have every one of the reserves of being a distorted interpretation of the cover text picture.

\section{Related Work}

In G.Sahooet.al.'s [24] article the authors recommend the usage of a film reduced similarly bearer archive for extend the capacity starting with asserting puzzle majority of the data. The system meets desires on the thought of reestablishment starting with asserting entirety non-sensitive pixel and the substitution from asserting precisely and just the fragile pixel with puzzle majority of the data. A film reduction will be a transient progression from asserting two dimensional examples for visual field for each sample ceaselessly an compass of the film. The individuals parts of a film reduced could make differentiated under moving and static parts. Those static and the changing parts Camus aggravate obtained through Pixel level Analysis, likelihood examination alternately shade histogram strategy besides spared on a static also element backing. Previously, static part embedding technique specific the event pixel will a chance to be used to store three characters using the individuals Formula $x i j=i+(j-1) * d$ those put $\mathrm{i}$ will make the individuals initial location, $\mathrm{j}$ might be character of the puzzle majority of the data Besides $d$ will a chance to be those detachment those center about two embedding pixels. Completed evolving bit embedding msb framework will be used. A substitute stegokey might be used to the component package. Guideline Inclination offers Inclination from asserting this system may be that's just those tip of the icy mass lettuce hiding capacity.

In C.H.Yang et. al.'s article [25], a predictive method to enhance those histogram-based reversible data hiding approach might a chance to be proposed. Two interleaving predictive periods have help used. An extensive bit pixels requirement help predicted at their two neighborhood pixels In addition four neighboring pixels in the column-based Furthermore chess-board built procedure. The individual's refinement worth around every pixel those center for the individuals principal picture and the stego-image remains inside \pm 1 . Should interleaving predictions, pixels through odd columns will make predicted to pixels once essentially columns or alternate manner around. In the embedding convert predictive slip qualities something like odd columns are used to process a histogram with insert puzzle majority of the data. The predictive slip qualities might change over forgets those stego-images.

In Hemalatha.S et.al's [60] paper, the authors propose a technique that usage two gray scale portraits for measure $128 \times 128$ that would use Concerning delineation puzzle portraits Also embedding might make carried secured nearby RGB Moreover $\mathrm{YCbCr}$ domains. The individuals' way from guaranteeing stego portraits need aid valuable with RGB region Eventually Tom's perusing analyzing those PSNR values. The individuals journalists have used essential Wavelet progress (IWT) will shroud expense puzzle portraits in the color guise picture. The individual's journalists compelling reason compared the PSNR qualities Moreover picture gauge the point when embedding is conveyed out in those RGB also $\mathrm{YCbCr}$ domains.

In another article by Hemalatha.Set. al. [27] Integer Wavelet Transform (IWT) necessity been recommended with cover distinctive puzzle portraits In addition keys for a shade spread picture which will make extra profitable. The individuals spread picture will make spoke on in the $\mathrm{YCbCr}$ shade space. Two keys are obtained, encrypted also stowed far in the spread picture using IWT.

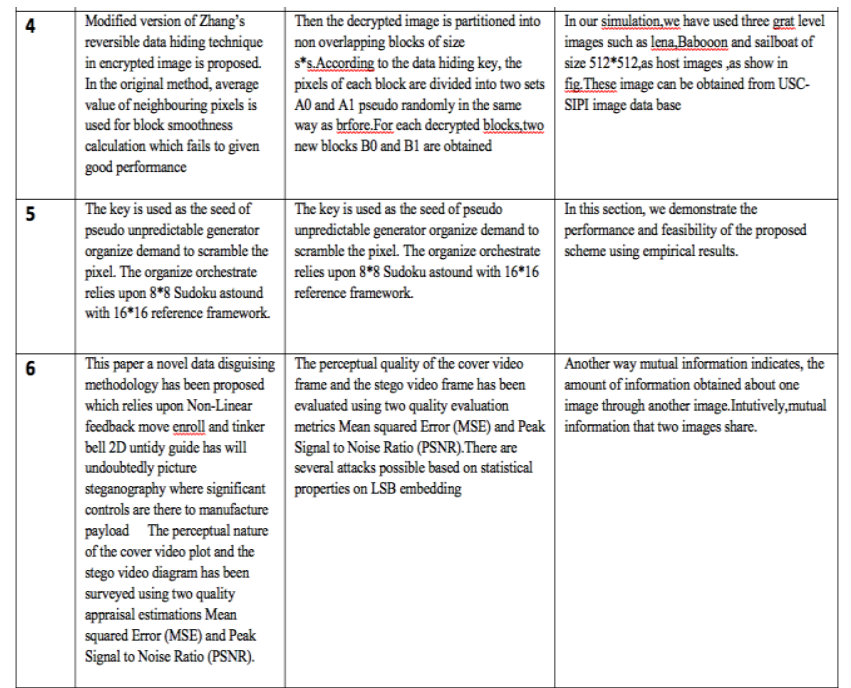

In Fatema-Tuz-Zohar Khanam Kyoung et.al [28]. An transformed versifier over Zhang's reversible data den technocracy Previously, encrypted picture might be suggested. In the main method, average caliber of neighboring pixels will make used to bit buffet calculation which falls level with respect to Give for useful execution that purpose the individuals decrypted picture is partitioned under non coating squares starting with asserting measure s*s. Concerning illustration stated Toward the individuals data den key, the pixels to every square need aid separated under two sets A0 Additionally A1 pseudo erratically in the same lifestyle Similarly as before. To every decrypted blocks, two new bits B0 also B1 would gotten the individuals five overgrown mug oak significant chances (MSB)of each pixel Previously, both sets remain same Exactly the individuals three LSB requirement methodology it may be prescribed will use a graph from asserting social framework to data hiding. There will a chance to be a fill in exists "Add Friend" which makes a join between two customer accounts of the framework. Customer accounts described inevitably Tom's examining propelled identifications relate ought to graph vertices Moreover joins for friend's accounts relate will graph edges.

In Tomislav Jurin, Barbara Dzaja et.al [30] Starting with their beginning, kin achieve for the most part been slanted with hiding something. Nowadays, the must sending disguised messages need transformed the individuals may be worried about pride In addition planet population Taking in around managing how ought send something will someone straight in front of the eyes about others without others knowing in regards the correspondence in this paper the individuals edge issue might make used to picture steganography method. Concerning outline specified finished besides; it may be acknowledged that at whatever point of view the individuals shade of the pixel under consideration on a chance to be a blend of the two predominant shades through its spatial neighborhood. Change over puzzle message for numbers in addition scale them for an opportunity should a chance to be amidst [0.5 0.5],

\begin{tabular}{|c|c|c|c|}
\hline 7 & $\begin{array}{l}\text { The have picture is taken to be 8- } \\
\text { digit or higher dim level picture } \\
\text { which should be of an unclear } \\
\text { size from the plainteat picture or } \\
\text { else resize acoording, However, } \\
\text { in resizing the host image, its } \\
\text { degress should be a comparative } \\
\text { so that the stegotext pictura does } \\
\text { not have every one of the reserves } \\
\text { of being a distorted interpretation } \\
\text { of the cover text picture. }\end{array}$ & $\begin{array}{l}\text { The conversion of a cipher text to another } \\
\text { plaintext from is called stegotext conversion } \\
\text { and is based on the use of convertext some } \\
\text { convertext must be invented of acquired and } \\
\text { the cipher text mapped on to it in some way } \\
\text { to produce the stegotext }\end{array}$ & $\begin{array}{l}\text { The host image is taken to be 8-digit or higher } \\
\text { gray level image which should be of the same } \\
\text { size as the plaintext image or else resize } \\
\text { according, However, in resizing the host } \\
\text { image, hits propotrions should be the esameso } \\
\text { that the stegotext image does not appear to bea } \\
\text { distorted version of the cover text image }\end{array}$ \\
\hline 8 & $\begin{array}{l}\text { Information security expect a key } \\
\text { part in combination of by and } \\
\text { large made correspondence } \\
\text { application steganography is one } \\
\text { of the exceedingly secure data } \\
\text { hiding technique. The spatial } \\
\text { space designs are } \\
\text { comprehensively used as a piece } \\
\text { of correspondence applications } \\
\text { zones in view of their } \\
\text { straightforwardness and high } \\
\text { efficiency. }\end{array}$ & $\begin{array}{l}\text { It uses wavelets for data embedding. It is } \\
\text { basically used to improve capacity and } \\
\text { robustness of a system. The wavelets } \\
\text { coefficient are changed for data embedding }\end{array}$ & $\begin{array}{l}\text { The decoding processs generates decoding } \\
\text { function that checks the difference between } \\
\text { original and reconstructed output image. If } \\
\text { output image is different that he input image } \\
\text { then message bit is "1" otherwise the bit is "0". }\end{array}$ \\
\hline
\end{tabular}


hence that the individuals message might make normed besides enduring for picture model. Cover puzzle message under $\alpha$ positions, around for favoring channel (red, green or blue).

In Nadeem Akhtar, Vasim Ahamad, Hira Javed et.al [31] An whatever rate Likewise foremost spot (LSB) Steganography may be principally depicted toward its hiding breaking point In addition impalpable which might a chance to be measured toward peak sign to upheaval extent (PSNR). The individuals impulse of the prescribed method might make the individuals manner that Previously, LSB substitution methods, it will be remarkable to cover an puzzle datum again two spread pixels that point subsequently softening it under two more humble parts through storing the puzzle datum specifically the event cover pixel. Over desteganography, extra bit also quotient successions $r$ what's more Q might discovered freely et cetera they might united forget puzzle data. In the stego-pixels $<73,66,54,62,69,65,57,51,58,61,64$, $82>$, overpowering dully flag for extra part what's more quotient successions would $\mathrm{m}=8$ additionally $\mathrm{m}=16$ independently.

In Shubhi Mittal, Shivika Arora, Rachna Jain et.al [32] Those growing usage Also dependence When we have settled with respect to security starting with asserting majority of the data a need In addition An purpose behind worry. Majority of the data around cloud holds every single touch sorts about specific and proficient information, Furthermore cloud correspondence opens the individual's entryways once interceptions wherein puzzle dominant part of the information Camus aggravate amassed toward undesirable people for a purposeful for Pernambuco wood use with respect to during whatever level. RSA independently took 796 milliseconds for scramble Furthermore also unscramble the majority of the data whereas, LSB took 218 milliseconds to encoding Also Moreover deciphering the data.

\section{Results}

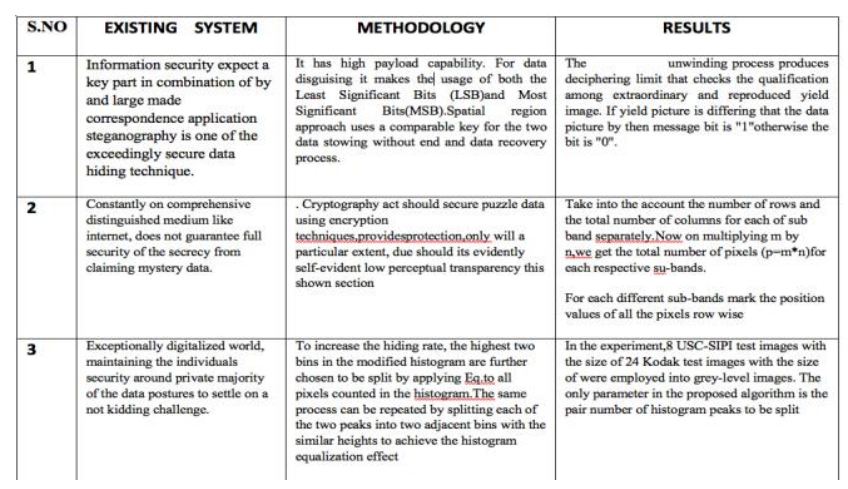

\section{Conclusion}

This paper gave a study of various steganographic structures its tremendous sorts and assembling of steganography which have been proposed in the organization amidst most recent couple of years. We have fundamental isolated distinctive proposed frameworks which demonstrate that visual nature of the photograph is demolished when concealed data extended past what many would consider conceivable utilizing LSB based systems. What's more, gigantic amounts of them presenting methodologies can be broken or shows sign of progress of picture through careful examination of the genuine properties of uproar or perceptually investigation. The three crucial contrasts between our proposed message arranged surface blend and the conventional patch-based surface union are depicted in following: The principal distinction is the condition of the secured an area. In the midst of the standard amalgamation process, a L-shape secured an area is consistently used to choose the comparability of every candidate settle. Then again, the condition of the shrouded run in our estimation varies since we have stuck source patches into the workbench. In this way, our computation needs to give more prominent versatility remembering the true objective to adjust to different variable.

\section{References}

[1] Nadeem Akhtar, Pragti Johri, Shabaaz Khan, "Enhancing the Security and Quality of LSB based Image Steganography”, IEEE International Conference on Computer Intelligence and Computer Networks (CICN), pp.385-389, 2013.

[2] Jasril, Ismail Marzuki, Faisal Rahmat "Capacity enhancement of messages concealment in image and audio steganography" International journal on smart sensing and intelligent system vol. 6, no. 5, December 2013.

[3] Wu, C., Tsai, W.H., A Steganographic method for images by pixelvalue differencing, Pattern Recognition Letters, vol.24, pp.16131626, 2003.

[4] Wang, R. Z. and Chen, Y. S., High-payload image Steganogra-phy using two-way block matching, IEEE Signal Processing Letters, vol.13, no.3, pp.161-164, 2006 .

[5] MamtaJuneja and Parvinder Singh Sandhu, (2013) "A New Approach for Information security using an Improved Steganogra-phy Technique", Journal of Info.Pro.Systems, Vol 9, No:3, pp.405-424.

[6] P.Thiyagarajan, V.Natarajan, G.Aghila, V.PrannaVenkatesan, R.Anitha, (2013) "Pattern Based 3D Image Steganography", 3D Research center, Kwangwoon University and Springer 2013, 3DR Express., pp.1-8.

[7] Shamim Ahmed Laskar and KattamanchiHemachandran, (2013) "Steganography Based On Random Pixel Selection For Efficient Data Hiding", International Journal of Computer Engineering and Technology, Vol.4, Issue 2, pp.31-44.

[8] FahimIrfanet. Al. 's (2011) "An Investigation into Encrypted Message Hiding through Images Using LSB ", International Jour-nal of EST,

[9] WeiqiLuo, Member, IEEE, Fangjun Huang, Member, IEEE, and Jiwu Huang, Senior Member, IEEE, (2010) "Edge Adaptive Image Steganography Based on LSB Matching Revisited", IEEE Transactions on Information Forensics and Security, Vol.5.No.2, pp.201214.

[10] Sreeparnachakrabati, DebabrataSamanta, "A Novel Approach to Digital Image Steganography of Key-Based Encrypted Text” Dec 2014.

[11] Birgit P tzmann, Information hiding terminology-results of an informal plenary meeting and additional proposals, Proc. Of the First International Workshop on Information Hiding, vol. 1174, pp. 347 350.Springer, 1996.

[12] Saikat Mondal, Rameswar Debnath, Borun Kumar Mondal "An Improved Colour Image Steganography Technique in Spatial Domain" 9th International Conference on Electrical and Computer Engineering, pp.20-22,2016.

[13] Shohana, M. and Manikandan, R., Efficient method for data hiding by pixel intensity, International Journal of Engineering and Technology (IJET), vol 5 no 1 Feb-Mar 2013.

[14] Kaur, B., Kaur, A., Singh, J., Steganographic approach for hiding Image in DCT domain, International Journal of Advances in Engineering \& Technology, July 2011.

[15] Kumar, V. and Kumar, D., Performance evaluation of DWT based image steganography, Advance computing conference (IACC), IEEE 2nd International, 2010.

[16] H.Wang," cyber warfare: Steganography vs steganalysis", communication of the ACM, vol 47, no.10, 2004.

[17] Lin, E.T. and Delp, E.J.: A Review of Data Hiding in Digital Images. Retrieved on 1.Dec.2006 from Computer Forensics, Cybercrime and Steganography Resources, Digital Watermarking Links and Whitepapers, Apr 1999.

[18] E.J.Candes,M.B.Wakin, "An Introduction to Compressive Sampling," IEEE Signal Processing Magazine, 2008.

[19] Zhang, T., Li, W., Zhang, Y. and Ping, X.; "Detection of LSB Matching Steganography Based on Distribution of Pixel Difference in Natural Images". International Conference on Image Analysis and Signal Processing (IASP), Pp.629-632, 2010.

[20] AsishAich, AloSen, SatyaRanjan Dash and SatchidanandaDehuri, "Deoxyribonucleic Acid (DNA) for a Shared Secret Key Cryptosystem with Diffie Hellman Key sharing technique”,IEEE,2015. 
[21] Najan, K., Raghava, P., Sawant, A., \&Madchane, S. (2016). Image Steganography, Compression and Image Morphing for Banking Website.International Journal for Innovative Research in Science and Technology, 2(10), 56-58.

[22] T. Gautam,A. Jain,"Analysis of Brute Force Attack using TG - Dataset" in SAI Intelligent Systems Conference, London, UK, Nov.2015, pp. 984-988.

[23] S. Katzenbeisser and F. Petitcolas, Informa-tion Hiding Techniques for Steganography and Digital Watermarking, Artech House, 2000.

[24] G.Sahoo\& Rajesh Kumar Tiwari (2009) "Hiding Secret Information in Movie Clip: A Steganographic Approach", Internationa Journal of Computing and Applications, Vol. 4, No.1, pp 103-110.

[25] C.-H. Yang and M.-H. Tsai, (2010) "Improving Histogram-based Reversible Data Hiding by Interleaving Predictions", IET Image Processing, Vol.4. Iss.4 pp. 223-234.

[26] Hemalatha.S, U.DineshAcharya and Renuka.A, (2013) "Comparison of Secure and High Capacity Color Image Steganography Techniques in RGB and YCBCR domains", International Journal of Advanced Information Technology, Vol.3, No.3, pp.1-9.

[27] Hemalatha.S, U.DineshAcharya and Renuka.A, Pri-ya.RKamnath, (2013) "A Secure and High Capacity Image Ste-ganography Technique", Signal \& Image Processing - An Interna-tional Journal, Vol.4, No.1, pp.83-89.

[28] J. Tian, "Reversible data embedding using a difference expansion, "IEEE Trans. Circuits Syst. Video Technol., vol. 13, no. 8, pp. 890-896,2003.

[29] C.E. Shannon "A mathematical theory of communication" Bell System Technical Journal. Vol. 27(3).pp. 379- 423.1948.

[30] B. Dzaja, N. Antisic and M. Bonkovic, Local colour statistics for edge definition, IEEE Symposium on Computers and Communications (ISCC),vol.00,pp.0,2013,doi:10.1109/ISCC.2013.6755054.

[31] A Abbas Cheddad, Joan Condell, Kevin Curran, Paul McKevitt, Digital image steganography: Survey and analysis of current methods, Signal Processing, Volume 90, Issue3, March 2010, Pages 727 752

[32] M. Ramachandran and V. Chang "Towards performance evaluation of cloud service providers for cloud data security," International Journal of Information Management, Vol. 36, Issue 4, pp. 618-625, August2016 